\title{
Metformin: Arguments for Maintaining its Position as First-Line Pharmacological Treatment in Type 2 Diabetes Mellitus
}

Authors:

${ }^{*}$ Coen DA Stehouwer ${ }^{1,2}$

1. CARIM School for Cardiovascular Diseases, Maastricht University, The Netherlands

2. Department of Internal Medicine, Maastricht University Medical Centre+, The Netherlands

*Correspondence to cda.stehouwer@mumc.nl

Disclosure: $\quad$ The author has declared no conflicts of interest.

Received: $\quad 31.08 .21$

Accepted: $\quad 29.09 .21$

Keywords: Glucagon-like peptide-1 receptor agonists (GLP-1RA), guidelines, metformin, pharmacological treatment, sodium-glucose co-transporter-2 inhibitors (SGLT2-i), Type 2 diabetes mellitus.

Citation: EMJ Diabet. 2021;9[1]:56-59.

\section{CASE}

A 62-year-old female was referred to her primary care physician for newly diagnosed Type 2 diabetes mellitus (T2DM). She was overweight (BMI: 29.1 $\mathrm{kg} / \mathrm{m}^{2}$ ) and had well-controlled hypertension and dyslipidaemia. She stopped smoking 5 months previously, after she had suffered a myocardial infarction. Her history was otherwise unremarkable. Glycated haemoglobin was $8.4 \%$ $(68 \mathrm{mmol} / \mathrm{mol})$; her estimated glomerular filtration rate was normal and there was no albuminuria. She was moderately physically active and tried to adhere to a healthy diet. She was not motivated to enter a weight-loss programme at this point. Her physician considered pharmacological treatment of her diabetes, especially as intensification of lifestyle changes in this patient would be difficult. How should this patient be advised?

'Good' versus 'less good' glycaemic control has been shown to reduce the incidence and progression of classic microvascular complications of diabetes (i.e., retinopathy, kidney disease, and polyneuropathy). These effects are widely held to be mostly independent of the way good glycaemic control is achieved, although this has not been formally demonstrated. However, good glycaemic control, at most, modestly reduces the incidence of macrovascular disease. Additionally, good glycaemic control has not convincingly been shown to reduce the incidence or progression of other complications of diabetes such as heart failure, late-life depression, and cognitive impairment, which may have mixed microvascular, macrovascular, and metabolic origins.

In this context, recent large cardiovascular and renal outcome trials have clearly shown that, in T2DM, sodium-glucose co-transporter-2 inhibitors (SGLT2i), and glucagon-like peptide-1 receptor agonists (GLP-1RA) reduce the incidence of cardiovascular events, heart failure, and kidney disease progression, especially in people at high cardiovascular risk. Additionally, such findings have been reported for multiple members of each drug class, notably for the SGLT2i empagliflozin, dapagliflozin, and canagliflozin; and for the GLPIRA liraglutide, semaglutide, albiglutide, and dulaglutide. ${ }^{1-3}$ It should be noted that the outcomes of these trials, even within one drug class, cannot be considered truly identical. The difficulty of deciding whether there is a 'class effect' is well illustrated by the VERTIS CV trial. This trial, which used ertugliflozin, did not show a reduction in major adverse cardiovascular outcomes, even 
though the participants in this trial were at high cardiovascular risk. ${ }^{4}$ Nevertheless, the fact that beneficial effects have been demonstrated for more than one drug in each class greatly increases confidence in the overall results.

Appropriately, the American Diabetes Association (ADA), together with the European Association for the Study of Diabetes (EASD) and the European Society of Cardiology (ESC), have responded to these developments by issuing strong recommendations for the use of SGLT2i and GLP-1RA in people with T2DM at high cardiovascular risk. ${ }^{1-3}$ Both guidelines recommend the use of these agents in people with T2DM and established atherosclerotic cardiovascular disease (CVD) or without established atherosclerotic CVD but deemed at high cardiovascular risk because of the presence of multiple other risk factors. Importantly, both guidelines recommend use of these agents regardless of the level of glycaemic control because these outcome trials did not target glycaemic control per se; because effects were by and large consistent across levels of glycaemia; and because effects appeared to be largely independent of the improvement of glycaemic control that is achieved by these agents.

However, a key difference between the recommendations by the ADA and the EASD on the one hand and the ESC on the other concerns the role of metformin. Briefly, the ADA and EASD continue to recommend metformin as first-line pharmacological treatment in people with T2DM, regardless of level of cardiovascular risk, whereas the ESC limits first-line use of metformin to people with T2DM with diabetes duration of up to 10 years and without other risk factors. $^{1-3}$ This paper will discuss the pros and cons of these diverging points of view.

In the trials on which the recommendations are based, metformin was usually prescribed as baseline therapy. ${ }^{1-3}$ Therefore, a strict interpretation of these trials would argue in favour of continuing using metformin as baseline therapy. Against this, trial results appeared similar in users and non-users of metformin, ${ }^{5}$ although this has not been evaluated in an appropriately designed (i.e., individual participant-level) meta-analysis. Non-users of metformin are a heterogeneous group ${ }^{6}$ as non-use may be related to intolerance (probably a major factor), as well as to (perceived) contraindications such as a history of heart failure or reduced glomerular filtration rate. Therefore, and especially with regard to metformin intolerance, the conclusion that, in people with T2DM, SGLT2i, and GLP-1RA have effects that are in general independent of the background use of metformin rests on the assumption that people who are prescribed metformin but cannot tolerate it will not differ importantly from other people with respect to the effects SGLT2i and GLP-1RA. As the biological basis of metformin intolerance is not well understood, this assumption is difficult to test outside a cardiovascular outcome trial.

Against maintaining metformin as a first-line therapy is the argument that the evidence base for metformin, insofar as it is derived from randomised controlled trials, is not very strong. Some trials did find that metformin reduced the incidence of cardiovascular events, 7,8 but a metaanalysis of randomised controlled trials found 13 trials reporting on just 2,079 individuals with T2DM allocated to metformin and a similar number to comparison groups. Participants were mainly white, aged 65 years or less, overweight or obese, and with poor glycaemic control. All outcomes, with the exception of stroke, favoured metformin, but none achieved statistical significance. ${ }^{9}$ The authors concluded that there remains uncertainty about whether metformin reduces risk of CVD in T2DM, and that this is mainly due to absence of evidence, ${ }^{9}$ which, of course, should not be equated with evidence of absence.

In contrast, observational data overwhelmingly support the use of metformin. For example, a systematic review found that metformin users with T2DM had significantly lower all-cause mortality compared with people without diabetes (hazard ratio [HR]: 0.93; 95\% confidence interval [Cl]: 0.88-0.99) or people with diabetes receiving non-metformin therapies (HR: $0.72 ; 95 \% \mathrm{Cl}: 0.65-0.80$ ), insulin (HR: 0.68; 95\% Cl: 0.63-0.75), or sulfonylurea (HR: 0.80; 95\% Cl: 0.66-0.97). Metformin users with T2DM also had a reduced incidence of cancer compared to people without diabetes (rate ratio: 0.94; 95\% Cl: 0.92-0.97) and of CVD compared with people with diabetes receiving non-metformin therapies (HR: 0.76; 95\% Cl: 0.660.87) or insulin (HR: 0.78; 95\% Cl: 0.73-0.83). ${ }^{10}$ 
The findings on cancer may be especially important. ${ }^{11,12}$ Although cancer is not traditionally considered a complication of diabetes, people with diabetes are more likely to develop liver, pancreatic, endometrial, gallbladder, kidney, colorectal, bladder, and breast cancer..$^{12}$ A recent investigation using a nationally representative primary care database found that, in 2018 , cancer had overtaken CVD as the leading cause of excess death associated with diabetes. ${ }^{11}$ Thus, any anti-cancer effect of metformin may be of considerable importance. Indeed, a Phase III randomised placebo-controlled trial showed that 1 year of treatment with metformin reduced the recurrence of colorectal cancer precursors in 151 individuals without diabetes, ${ }^{13}$ suggesting that metformin might have chemopreventive effects against cancer. Metformin might influence tumourigenesis, both indirectly, through the systemic reduction of insulin levels, and directly, via induction of the adenosine monophosphateactivated protein kinase (AMPK) pathway and inhibition of the mechanistic target of rapamycin (mTOR) pathway.14,15 These effects obviously require further investigation, and a number of trials to test the anti-cancer effects of metformin are ongoing. ${ }^{15}$

In an ageing population, a further important consideration is metformin's potential preventive effects on cognitive impairment. Compared with normal glucose metabolism, T2DM is associated with a subtly but measurably worse cognitive performance and additionally with a 1.5-times increased risk of dementia.16,17 Observational studies have quite consistently shown that use of metformin is associated with reduced risk of cognitive impairment and dementia. ${ }^{15}$ These effects may again be related to adenosine monophosphate-activated protein kinase activation, through which metformin mimics the imbalance between energy supply and demand seen in fasting and exercise, thus activating pathways that reduce cellular stress. ${ }^{15}$
So how should the patient in the vignette above be advised? Metformin is a time-honoured option that may reduce CVD, cancer, and cognitive impairment. However, the cardiovascular effects of metformin remain a matter of controversy, and additional effects of metformin must be considered unproven. Additionally, starting with metformin monotherapy would deny the patient the cardiovascular benefits of a GLP-1RA or an SGLT2i for an undefined period of time. Conversely, starting treatment with a GLP-1RA or an SGLT2i would deny the patient any beneficial effects of metformin and, additionally, expose her to the unproven assumption that the effects of a GLP-1RA or an SGLT2i in T2DM are similar regardless of the use of metformin. Paradoxically, if this patient had presented with a glycated haemoglobin of $7.4 \%(57 \mathrm{mmol} / \mathrm{mol})$, while having been treated with metformin for 3 months, further treatment advice (i.e., continue metformin and add a GLP-1RA or an SGLT2i) would not be controversial. Further treatment advice would also not be controversial if, in this scenario, the patient had presented with a glycated haemoglobin of $6.8 \%(51 \mathrm{mmol} / \mathrm{mol})$, because, as mentioned before, the guidelines recommend use of a GLP-1RA or an SGLT2i regardless of level of glycaemic control.

Therefore, this patient should be advised to start combination treatment with metformin and a GLP-1RA or an SGLT2i. This recommendation is fully in line with the way GLP-1RA and SGLT2i were used in the trials on which the guidelines are based. Additionally, there is the possibility that such combination treatment will provide greater and more durable long-term benefit with respect to glycaemic control, as demonstrated for the combination of metformin and a dipeptidyl peptidase-4 inhibitor as compared with metformin monotherapy. ${ }^{18}$ Finally, metformin may have anti-cancer effects and slow down cognitive impairment, which are exciting potential additional benefits.

\section{References}

1. Davies MJ et al. Management of hyperglycemia in Type 2 diabetes, 2018. A consensus report by the American Diabetes Association (ADA) and the European Association for the Study of Diabetes (EASD). Diabetes Care. 2018;41(12):2669-701.
2. Buse JB et al. 2019 update to: management of hyperglycemia in Type 2 diabetes, 2018. A consensus report by the American Diabetes Association (ADA) and the European Association for the Study of Diabetes (EASD). Diabetes Care. 2020;43(2):487-93.
3. Cosentino F et al. 2019 ESC Guidelines on diabetes, pre-diabetes, and cardiovascular diseases developed in collaboration with the EASD. Eur Heart J. 2020;41:255-323.

4. Cannon $\mathrm{CP}$ et al. Cardiovascular outcomes with ertugliflozin in 
Type 2 diabetes. N Engl J Med. 2020;383(15):1425-35.

5. Marx $\mathrm{N}$ et al. Guideline recommendations and the positioning of newer drugs in Type 2 diabetes care. Lancet Diabetes Endocrinol. 2021;9(1):46-52.

6. Zaccardi $F$ et al. First-line treatment for Type 2 diabetes: is it too early to abandon metformin? Lancet. 2020;396(10264):1705-7.

7. Holman RR et al. 10-year followup of intensive glucose contro in Type 2 diabetes. $\mathrm{N}$ Engl J Med. 2008;359(15):1577-89.

8. Kooy $A$ et al. Long-term effects of metformin on metabolism and microvascular and macrovascular disease in patients with Type 2 diabetes mellitus. Arch Intern Med. 2009;169(6):616-25.

9. Griffin SJ et al. Impact of metformin on cardiovascular disease: a metaanalysis of randomised trials among people with Type 2 diabetes. Diabetologia. 2017;60(9):1620-9.

10. Campbell JM et al. Metformin reduces all-cause mortality and diseases of ageing independent of its effect on diabetes control: a systematic review and meta-analysis. Ageing Res Rev. 2017:40:31-44

11. Pearson-Stuttard $\mathrm{J}$ et al. Trends in predominant causes of death in individuals with and without diabetes in England from 2001 to 2018: an epidemiological analysis of linked primary care records. Lancet Diabetes Endocrinol. 2021;9(3):165-73.

12. Song $M$. Cancer overtakes vascular disease as leading cause of excess death associated with diabetes. Lancet Diabetes Endocrinol. 2021:9(3):131-3.

13. Higurashi T et al. Metformin for chemoprevention of metachronous colorectal adenoma or polyps in post-polypectomy patients without diabetes: a multicentre double-blind, placebo-controlled, randomised phase 3 trial. Lancet Oncol. 2016;17(4):475-83.

14. Pernicova I, Korbonits $M$. Metformin--mode of action and clinical implications for diabetes and cancer. Nat Rev Endocrinol. 2014;10(3):143-56.
15. Giaccari A et al. Metformin benefits: another example for alternative energy substrate mechanism? Diabetes Care. 2021;44(3):647-54

16. Geijselaers SLC et al. The role of hyperglycemia, insulin resistance, and blood pressure in diabetes-associated differences in cognitive performanceThe Maastricht Study. Diabetes Care. 2017;40(11):1537-47.

17. van Sloten TT et al. Cerebral microvascular complications of Type 2 diabetes: stroke, cognitive dysfunction, and depression. Lancet Diabetes Endocrinol. 2020;8(4):325-36.

18. Matthews DR et al. Glycaemic durability of an early combination therapy with vildagliptin and metformin versus sequential metformin monotherapy in newly diagnosed Type 2 diabetes (VERIFY): a 5-year, multicentre, randomised, double-blind trial. Lancet. 2019;394(10208):1519-29. 